\title{
COVID-19-Betroffenheit in der vollstationären Langzeitpflege
}

Raphael Kohl, Kathrin Jürchott, Christian Hering, Annabell Gangnus, Adelheid Kuhlmey und Antje Schwinger

\section{Inhaltsverzeichnis}

1.1 "Die Pandemie hat uns kalt erwischt" - 4

1.2 COVID-19 und Hochbetagte - 6

1.3 Deskription des Pandemiegeschehens in Pflegeheimen während der Pandemiephase März bis Mai 2020 - 8

1.3.1 Sterblichkeit von Pflegebedürftigen in vollstationärer Pflege -8

1.3.2 Pflegeheimbewohnende mit COVID-19 im Krankenhaus - 12

1.3.3 Auswirkung der COVID-Pandemie auf die Krankenhausversorgung der Pflegeheimbewohnenden - 14

1.4 Diskussion und Ausblick - 16

Literatur - 18 


\section{- Zusammenfassung}

Die Corona-Pandemie deckt die Schwächen des stationären Pflegesystems - schon vor der Pandemie waren diese abzusehen - nun schonungslos auf. Der vorliegende Beitrag zeichnet basierend auf Abrechnungsdaten der AOKPflege- und Krankenkassen ein Abbild dieser Krisensituation für die erste Pandemiewelle von März bis Mai 2020. Aufgezeigt wird die Sterblichkeit von vollstationär Pflegebedürftigen, die in der 15. Kalenderwoche $20 \%$ höher als im Mittel der Vorjahre lag und dann bis zur 20. Kalenderwoche wieder auf den Durchschnitt der Vorjahre abfiel. Mit Blick auf COVID-Erkrankte entfiel jeder fünfte COVID-Krankenhausaufenthalt und fast jeder dritte COVID-Todesfall im Krankenhaus auf einen vollstationär versorgten Pflegebedürftigen. Die Ergebnisse zum massiven Rückgang der Krankenhausaufnahmen von Pflegebedürftigen aus den Heimen zwischen der 10. und 12. Kalenderwoche des Jahres 2020 um rund ein Drittel werfen zudem ein erstes Schlaglicht auf mögliche sekundäre Folgen für die Gesundheitsversorgung während der ersten Pandemiewelle. Der Beitrag legt damit erste Befunde aus dem Kontext des durch den Spitzenverband der Gesetzlichen Krankenversicherung (GKV-SV) geförderten Forschungsprojektes Covid-Heim vor, das von der Charité - Universitätsmedizin Berlin in Kooperation mit dem Wissenschaftlichem Institut der AOK (WIdO) durchgeführt wird. Ziel des Projektes ist eine möglichst umfassende Beschreibung der Situation der Heimbewohnerinnen und -bewohner, ihrer Angehörigen und der Pflegekräfte, um so eine belastbare Evidenz für Veränderungen im Versorgungssetting der stationären Langzeitpflege zu erhalten.

The Corona pandemic highlights the deficits of the long-term care system in Germany. Based on claims data from the AOK nursing and health insurance funds, the article describes the situation during the first wave of the pandemic from March to May 2020. It shows the mortality of people in need of long-term care, which in the 15th calendar week was $20 \%$ higher than the average of previous years and then falls back to the average of previous years by the 20th calendar week. Every fifth COVID hospitalisation and almost every third COVID death in hospital was attributable to a nursing home resident. The massive decrease in hospital admissions of nursing home residents from the 10th to the 12th calendar week of 2020 by about one third raises questions regarding possible secondary consequences for health care during the first wave of the pandemic. The article presents the first findings from the context of the Covid-Heim research project funded by the German National Association of Statutory Health Insurance (GKV-SV), which is being carried out by the Charite Universitätsmedizin Berlin in cooperation with the AOK Research Institute (WIdO). The aim of the project is to describe the situation of nursing home residents, their relatives and the nursing staff as comprehensively as possible and to conclude which changes are necessary concerning the provision of long-term care in nursing homes.

\section{1 „Die Pandemie hat uns kalt erwischt"1}

Im März 2020 stiegen die Infektionen mit dem neuartigen SARS-CoV-2-Coronavirus (COVID-19) rasant. Ein dynamisches Pandemiegeschehen, das wirtschaftliche, politische, bildungsbezogene und in besonderem Maße gesundheitliche Auswirkungen hatte, zeichnet sich bis heute fort. Als politische Konsequenz wurden zur Senkung der Infektionsgefahr und Unterbrechung des exponentiellen Wachstums der Infektionszahlen Infektionsschutzmaßnahmen in vielen gesellschaftlichen Bereichen erlassen, so auch in Pflegeeinrichtungen der vollstationären Pflege. Der Grundbaustein dafür wurde mit dem Bevölkerungsschutz-

1 Keller (2020). 
gesetz $^{2}$ gelegt. Dabei sind weitreichende Änderungen am bestehenden Infektionsschutzgesetz (ifSG) und am fünften Sozialgesetzbuch (SGB V) vorgenommen worden. So wurden im März 2020 in den stationären Pflegeheimen im Rahmen des „Lockdown“ - ex abrupto Maßnahmen zur Eindämmung der Pandemie in Form von Schließungen, Auferlegung von Besuchsverboten und Zugangsregelungen erlassen sowie soziale Angebote aufgehoben. Nahezu übergangslos waren die Pflegeeinrichtungen dazu gezwungen, die weggefallenen Versorgungsstrukturen bestmöglich zu kompensieren. Erhöhte Krankenstände haben die Arbeitsbedingungen des Pflegepersonals in der ersten Welle weiter erschwert (Drupp et al. im gleichen Band) und die Situation hat insgesamt zu einer hohen psychischen Belastung bei ihnen geführt (Hower et al. 2020). Aber auch für die Pflegeheimbewohnenden bestehen ungeachtet der gesundheitlichen Folgen einer SARS-CoV-2-Infektion massive Auswirkungen auf die soziale Teilhabe und damit das Wohlbefinden infolge der Schutzmaßnahmen (Schrappe et al. 2020b; DGP 2020; Kessler et al. 2020; Räker et al. im gleichen Band). Im Vergleich zu der ersten Welle der Corona-Pandemie, in der Versorgung und Teilhabe der Bewohnenden durch die Schutzmaßnahmen in einem schwer tolerierbaren Ausmaß beeinträchtigt waren (Kessler et al. 2020), wurde während der zweiten Welle stärker darauf geachtet, Versorgungsstrukturen aufrechtzuerhalten und zumindest grundlegende Kontakte zuzulassen. So sind Mitte Dezember 2020 verfügbare Schutzmasken (FFP2-Masken), vom Bund bezahlte Antigen-Schnelltests, verpflichtende Testungen mehrmals pro Woche für das Pflegepersonal und der Nachweis eines Negativtests für die Besuchenden verordnet worden (Bund-Länder-Beschlüsse 2020). Ab Januar 2021 konnte alsdann mit den ersten Impfungen sowohl der Bewohnenden als auch des Personals in den Heimen begonnen werden, sodass

2 Das erste Gesetz zum Schutz der Bevölkerung wurde am 27. März 2020, das zweite am 25. Mai 2020 und das dritte am 18 . November 2020 erlassen. mit Stand Ende März von einer weitestgehenden Durchimpfung der Pflegeheimbewohnenden auszugehen ist. ${ }^{3}$ Dennoch ist der Arbeitsund Lebensalltag in deutschen Pflegeheimen weiterhin von der Pandemie geprägt. Beispielsweise gelten nach den erfolgten Impfungen weiterhin die strikten Besuchsbeschränkungen in den Pflegeeinrichtungen - zulasten der Bewohnenden und deren Angehörigen wie auch des Pflegepersonals. Die bisher bestehenden Hygiene-, Schutz- und Testkonzepte sollen weiterhin konsequent umgesetzt werden. ${ }^{4}$

Die Dialektik zwischen Schutz vor Infektion und Wahrung der Menschenwürde und Autonomie der Alten- und Pflegeheimbewohnenden stellt eine stetige Herausforderung dar und wirft Fragen auf: Wie können sie prospektiv besser geschützt werden, ohne die Teilhabe zu gefährden? Welche Einflussfaktoren stehen womöglich im Zusammenhang mit der ohnehin schwierigen Versorgungssituation in den Pflegeheimen? Welche Maßnahmen und Strukturen in Pflegeeinrichtungen waren zur Infektionseindämmung wirksam? Wie gestaltet(e) sich die soziale Lebensund Versorgungssituation der Heimbewohnenden sowie des Pflegepersonals während der Pandemie deutschlandweit? Diese und weitere Schwerpunkte werden derzeit im Rahmen eines durch den GKV-Spitzenverband geförderten Forschungsprojekts - Covid-Heim - der Charité - Universitätsmedizin Berlin in Kooperation mit dem Wissenschaftlichen Institut der AOK (WIdO) untersucht. Ziel ist ein dezidiertes Bild der Situation der hochbetagten Bewohnenden in deutschen Pflegeheimen zu erhalten, um damit eine Bewertung der ge-

3 Digitales Impfquotenmonitoring zur COVID-19Impfung des RKI; Durchgeführte Impfungen bundesweit und nach Bundesland bis einschließlich 19.03.2021; Zugriff unter $>$ https://www.rki.de/DE/ Content/InfAZ/N/Neuartiges_Coronavirus/Daten/ Impfquoten-Tab.html.

4 Siehe z.B. Auszug aus der Zwölften Bayerischen Infektionsschutzverordnung (05.03.2021), > https:// www.verkuendung-bayern.de/baymbl/2021-171/, sowie auch die Ergebnisse der Videokonferenz der Bundeskanzlerin mit den Regierungschefinnen und Regierungschefs der Länder am 22. März 2021. 
troffenen Maßnahmen empirisch unterlegen zu können und zugleich einen Beitrag zur Diskussion perspektivischer Handlungsempfehlungen zu leisten. Die vorliegenden Analysen zeichnen basierend auf Abrechnungsdaten der AOK-Pflege- und Krankenkassen das Abbild einer Krisensituation für die erste Pandemiewelle von März bis Mai 2020. Nach einem kurzen Überblick zu COVID bei Hochbetagten werden für die erste COVID-Welle die allgemeine Sterblichkeit der Pflegeheimbewohnenden - d.h. die sogenannte Übersterblichkeit ohne direkten COVID-Bezug - und die Sterblichkeit der im Krankenhaus behandelten COVID-Erkrankten aus Pflegeheimen aufgezeigt. Ebenso wird am Beispiel der generell veränderten Krankenhausbehandlung der Pflegeheimbewohnenden ein erstes Schlaglicht auf potenziell sekundäre Folgen der Pandemie hier die gesundheitliche Versorgung der Pflegeheimbewohner - geworfen. Das Fazit greift die aufgeworfenen Problemfelder auf und diskutiert mögliche Ableitungen für künftige Forschungsvorhaben, u. a. mit Blick auf das laufende Forschungsprojekt Covid-Heim.

\subsection{COVID-19 und Hochbetagte}

Das neuartige Coronavirus trat das erste Mal im Dezember 2019 in Wuhan (China) in Erscheinung (Buonsenso et al. 2020), die dadurch ausgelöste Erkrankung wurde nach Identifizierung des Erregers von der Weltgesundheitsorganisation (WHO) als COVID-19 (Coronavirus-Krankheit-2019) bezeichnet (Asim et al. 2020). Zu den häufigsten Symptomen zählen Husten (40\%), Fieber (27\%), Schnupfen (29\%) sowie eine Störung des Geruchs- und/oder Geschmackssinns (22\%). Bei etwa 1\% der Fälle entwickelt sich im Verlauf der Erkrankung eine Pneumonie (RKI 2021a). Eine Ansteckung mit dem Virus findet hauptsächlich durch respiratorische Aufnahme von virushaltigen Aerosolen in der Luft durch beispielsweise Husten, Niesen, Sprechen oder auch Singen statt (RKI 2021a). Nachdem sich das Virus von China aus in hohem Tempo im Rest der Welt verbreitete, erklärte die WHO am 11. März 2020 den weltweiten Ausbruch von COVID-19 offiziell zur Pandemie (Cucinotta und Vanelli 2020). Zu diesem Zeitpunkt gab es in Deutschland 1.567 laborbestätigte SARS-CoV-2-Fälle sowie drei Todesfälle, die mit dem Virus assoziiert waren (RKI 2020a). Kurze Zeit später trat der erste „Lockdown“ in Kraft, um einen weiteren exponentiellen Anstieg der Infektionszahlen zu unterbinden.

Seit Beginn der Pandemie haben sich in Deutschland mehr als 2,5 Mio. Menschen infiziert, rund 75.000 sind mit oder an COVID-19 verstorben (Stand 20.03.2021; RKI 2021b). Hospitalisiert werden gemäß deutschem Meldesystem ca. $10 \%$ aller Infizierten (RKI 2021a). Der Fall-Verstorbenen-Anteil oder CFR (engl. case fatality rate) liegt für Menschen bis 50 Jahre bei $0,1 \%$ und nimmt mit dem Alter der Infizierten sukzessive $\mathrm{zu}$, sodass dieser für Infizierte ab einem Alter von 80 Jahren bei über $10 \%$ liegt (RKI 2021a). Bereits für die erste COVIDWelle konnte mit Hilfe von AOK-Abrechnungsdaten aus dem Zeitraum 26. Februar bis 19. April 2020 mit insgesamt 10.021 hospitalisierten COVID-19-Patientinnen und Patienten gezeigt werden, dass besonders hochbetagte Menschen ein erhöhtes Risiko für einen schweren oder tödlichen Verlauf einer COVID-19-Erkrankung aufweisen (Karagiannidis et al. 2020). Die hospitalisierten $\mathrm{Pa}$ tienten in diesem Zeitraum waren im Durchschnitt 68,3 Jahre alt. Insgesamt wurden $17 \%$ der Infizierten in Kliniken maschinell beatmet. Die Sterberate bei beatmeten Patienten in der Alterskohorte ab 80 Jahren lag bei $72 \%$. Insgesamt verstarben ca. $22 \%$ aller Patienten, die in der ersten Welle mit oder aufgrund einer COVID-19-Infektion in einer Klinik behandelt wurden. Die erhöhte Vulnerabilität der hochbetagten Menschen gegenüber COVID-19 geht unter anderem auf Veränderungen des Immunsystems im Alter und deren häufige Multimorbidität zurück (Mueller et al. 2020; Iaccarino et al. 2020). Assoziiert sind 
nach einem aktuellen systematischen Review des RKI u. a. folgende Risikofaktoren für einen schweren Krankheitsverlauf mit dem Endpunkt Mortalität: Herz-Kreislauf-Erkrankungen (Arrhythmie, Vorhofflimmern, Herzinsuffizienz, Koronare Herzkrankheit (KHK)), Diabetes mellitus, Übergewicht, chronische Nierenerkrankungen, schwere chronische Lungenerkrankungen (z. B. COPD), Demenz sowie Krebs- und Immunerkrankungen (RKI 2021c).

Nur für rund 213.000 Fälle (d.h. für insgesamt $8 \%$ des Infektionsgeschehens) sind in den Meldedaten des RKI Informationen enthalten, ob die Betroffenen in einer im Sinne des Infektionsschutzgesetzes relevanten Einrichtung betreut oder untergebracht sind. Lediglich 120.000 der entsprechenden gemeldeten Fälle beziehen sich auf Einrichtungen im Sinne des $\S 36$ IfSG, d.h. Pflegeeinrichtungen, Obdachlosenunterkünfte, Einrichtungen zur gemeinschaftlichen Unterbringung von Asylsuchenden, sonstige Massenunterkünfte und Justizvollzugsanstalten. Eine Differenzierung nach Einrichtungsart ist wiederum nur für rund 70.000 Fällen erfolgt, 64.000 Fälle wurden aus Pflegeheimen gemeldet. Der Anteil verstorbener Bewohnender wird mit $18 \%$ ausgewiesen (Stand 20.03.2021; RKI 2021b). Deutlich wird, dass dies lediglich den Mindestanteil darstellt, da es derzeit kein dezidiertes System zur Erfassung von COVID-19-Infektionen und -Todesfällen in Altenpflegeheimen gibt.

Neben der Bedrohung durch COVID-19 selbst wurde auch vor sekundären Folgen des „Lockdown“ für die hochbetagte Klientel in den Pflegeheimen gewarnt (Schrappe et al. 2020b). Soziale Deprivation und damit einhergehende Isolations- und Einsamkeitsgefühle sowie die sich verändernden Versorgungsroutinen (z. B. das Tragen von Schutzausrüstung durch das Pflegepersonal) können sowohl psychische als auch physische Folgen haben, denn Sozialbeziehungen sowie ein regelmäßiger sozialer Austausch sind bedeutsam für das subjektive Wohlergehen und wirken präventiv gegenüber depressiven Symptomatiken älterer
Menschen (Holt-Lunstad et al. 2010). Studien weisen diesbezüglich auf einen erhöhten Grad an Teilnahmslosigkeit, Agitiertheit und Ängsten während der COVID-19-Pandemie bei Hochbetagten mit Demenz hin, was durch Besuchseinschränkungen und die Einschränkung des Kontakts zum Pflegepersonal in den Altenheimen ausgelöst wird (Simonetti et al. 2020, Panagiotou et al. 2021). Darüber hinaus ist bisher wenig bekannt über die gesundheitlichen Sekundärfolgen der Pandemie bei Pflegeheimbewohnenden, die aus den Lockdown-bedingten veränderten Versorgungsangeboten resultieren. Mostert et al. (2021) zeigen auf, dass während der ersten COVID-Welle - und abgeschwächt auch in der zweiten - bezogen auf die Gesamtbevölkerung ein deutlicher Rückgang der Krankenhausaufnahmen zu beobachten war. Das Leistungsspektrum hat sich hin zu operativen Fälle verschoben und auf Fälle mit höherer Schwere konzentriert. Dabei zeigen sich aber auch Fallzahleinbrüche bei Notfallindikationen wie Herzinfarkt und Schlaganfall oder auch bei Krebsoperationen. Drogan et al. (2020) belegen beispielsweise für den Schlaganfall, dass während der ersten COVIDWelle neben einer deutlichen Fallzahlreduktion im Krankenhaus eine signifikant erhöhte 30-Tage-Sterblichkeit und auch eine absolute Erhöhung der Sterblichkeit zu beobachten war. Es besteht aus Sicht von Mostert et al. (2021) umfänglicher Forschungsbedarf zu den Auswirkungen der pandemiebedingten Veränderungen des Leistungsspektrums auf die gesundheitliche Versorgung und grundsätzlich zu der Frage, inwieweit die Veränderungen eine Fokussierung auf das originär behandlungsbedürftige Kerngeschäft bedeuten, d. h. Rückgänge dadurch erklärt werden können, dass nicht zwingend stationär behandlungsbedürftige Fälle nicht in Krankenhäuser aufgenommen wurden. Auch für die Inanspruchnahme vertragsärztlicher und vertragspsychotherapeutischer Leistungen bezogen auf die $\mathrm{Ge}$ samtbevölkerung ist über alle Facharztgruppen hinweg ein deutlicher Rückgang der Behandlungsfälle zu beobachten (Mangiapane et al. 2021). 
Vor dem Hintergrund dieser Erkenntnisse wird im Folgenden neben der allgemeinen Sterblichkeit der Pflegeheimbewohnenden während der ersten Welle die COVIDassoziierte Sterblichkeit im Krankenhaus, aber auch die generelle Krankenhausversorgung der Heimbewohnenden untersucht.

\subsection{Deskription des} Pandemiegeschehens in Pflegeheimen während der Pandemiephase März bis Mai 2020

\subsubsection{Sterblichkeit von Pflegebedürftigen in vollstationärer Pflege}

Die für das Studienziel anonymisierten Routinedaten der Jahre 2015 bis 2020 - jeweils bezogen auf die Monate Januar bis Juni - umfassen im Jahresschnitt zwischen 380.000 und 400.000 Pflegebedürftige im Alter von 60 Jahren und älter in vollstationären Pflegeheimen. Der Anteil Männer lag 2015 bei $27 \%$ und ist bis 2020 leicht auf $29 \%$ gestiegen. Die Alterszusammensetzung der betrachteten Kohorten ist mit durchschnittlich rund 80 Jahren bei den Männern und rund 86 Jahren bei den Frauen über diesen Zeitraum konstant geblieben. Für alle Versicherten, die je Kalenderwoche (KW) für mindestens einen Tag Leistungen nach $\S 43$ SGB XI bezogen, wurde analysiert, ob diese in der jeweiligen Kalenderwoche verstorben waren, und zwar unabhängig vom Ort - also beispielsweise, ob sie im Krankenhaus oder im Pflegeheim verstarben - und unabhängig von der Ursache des Versterbens. Die Sterberaten bewegten sich in den ersten Jahreshälften von 2015 bis 2020 in der Größenordnung zwischen 0,5 und $1,2 \%$, d.h. 5-12 von 1.000 Pflegeheimbewohnenden versterben je Woche (- Abb. 1.1). Deutlich hervor treten die Ster-

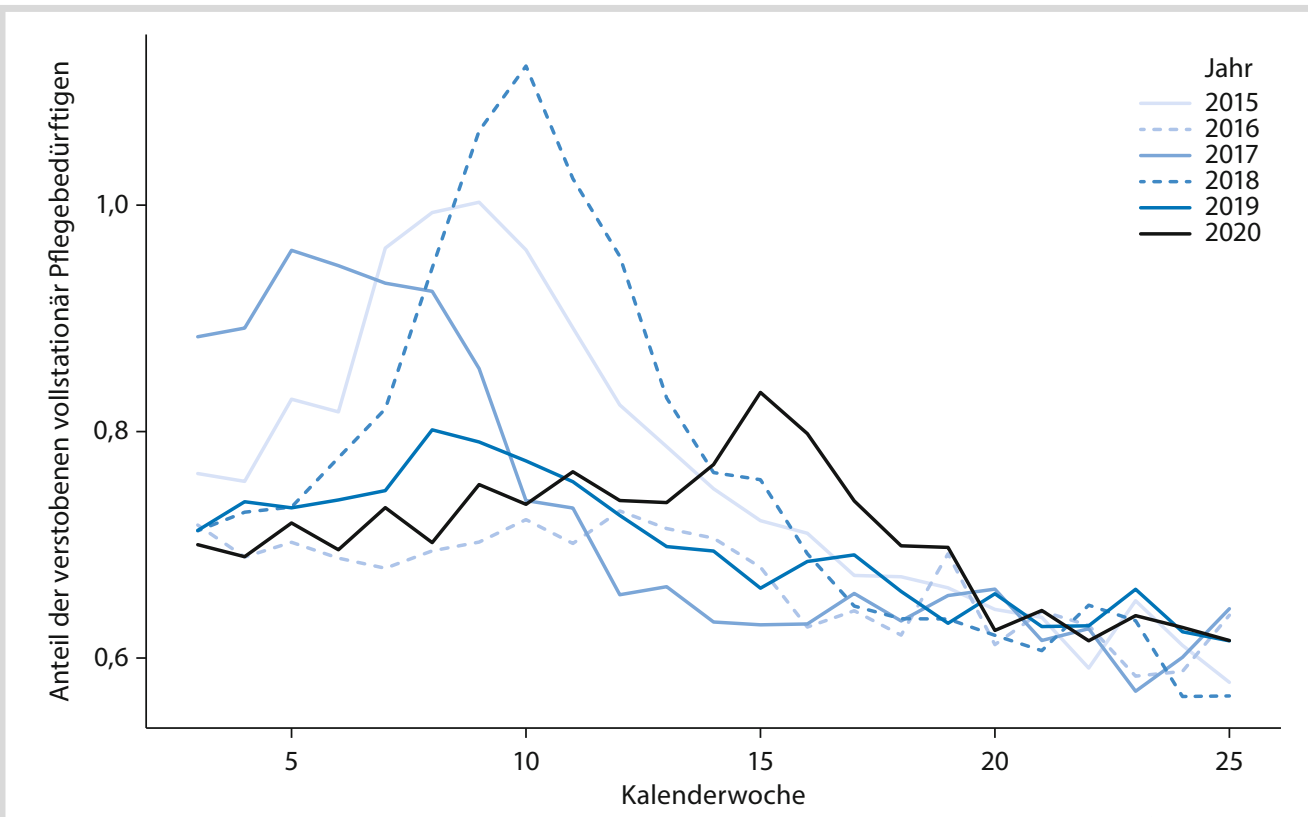

- Abb. 1.1 Sterblichkeit von vollstationär Pflegebedürftigen im Jahresvergleich 2015-2020, in \%. (Quelle: AOK-Routinedaten 2015-2020) 


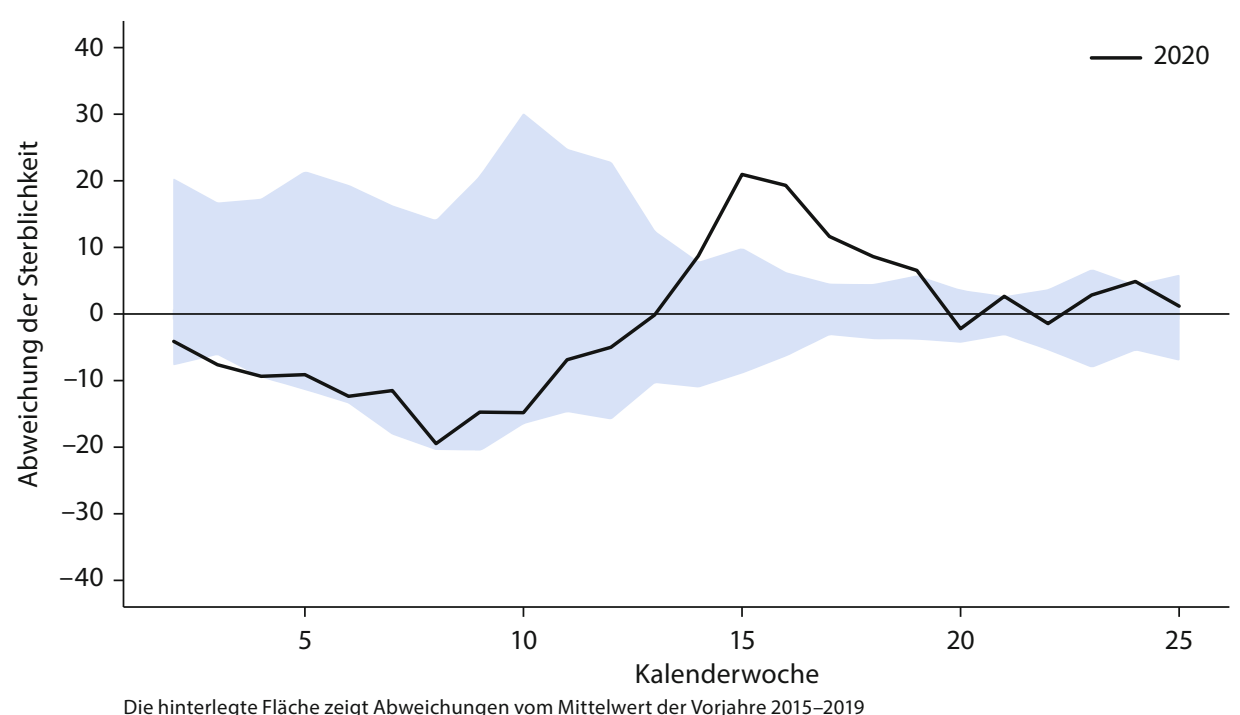

Pflege-Report 2021

- Abb. 1.2 Sterblichkeit von vollstationär Pflegebedürftigen im Jahr 2020 im Vergleich zum Mittelwert der Vorjahre 2015-2019, in \%. (Quelle: AOK-Routinedaten 2015-2020)

beraten der Jahre 2015, 2017 und 2018, welche die des Jahres 2020 übersteigen. Die beobachteten Ausschläge verweisen auf die durch das Surveillance-System des $\mathrm{RKI}^{5}$ aufgezeigten Grippewellen. Entsprechende kausale Zusammenhänge wurden im Rahmen des hier vorgelegten Beitrags jedoch nicht weiter untersucht.

- Abb. 1.2 zeigt die prozentuale Abweichung der Sterberate aus dem Jahr 2020 vom Mittelwert der Vorjahre 2015 bis 2019. Nachdem in der eigentlichen Grippesaison im Vergleich zu den Vorjahren weniger Pflegeheimbewohnende pro Woche verstarben, kehrte sich dieses Bild ab der 14. Kalenderwoche um. In

5 Zur ganzjährigen Beobachtung akuter Atemwegsinfektionen hat das RKI unter dem Namen GrippeWeb ein Online-Überwachungsinstrument etabliert, über das die für die Bevölkerung in Deutschland geschätzten wöchentlichen Raten akuter grippeähnlicher Erkrankungen (ILI) ausgewiesen werden. Für die Grippesaisons siehe beispielsweise $>$ https:// grippeweb.rki.de/Content/GrippeWeb_Kapitel_ Influenza_Saisonbericht_2017_18.pdf. der 15. Kalenderwoche (6. bis 12. April) also rund drei Wochen nach Start des Lockdowns ab 16. März - lag der Anteil der Verstorbenen um $20 \%$ höher, als es im Mittel der Vorjahre der Fall war, und sank dann bis zur 20. Kalenderwoche (11. bis 17. Mai) wieder auf den Durchschnitt der Vorjahre. Der beobachtete Verlauf deckt sich mit der für die Gesamtbevölkerung beobachteten Entwicklung (• Abb. 1.3).

Die Sterblichkeit unterscheidet sich zwischen den Altersgruppen: Fast $40 \%$ (37,3-39,1\%) der Todesfälle bei Pflegeheimbewohnenden entfielen in den Jahren 2015 bis 2019 auf die Gruppe der über 90-Jährigen, ein weiteres Viertel $(24,7-26,1 \%)$ auf die 85 - bis 89-Jährigen und zwischen 17,1 und 18,5\% auf

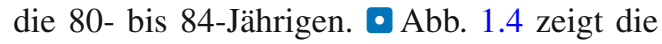
prozentuale Abweichung der Sterberate aus dem Jahr 2020 zum Mittelwert der Vorjahre 2015 bis 2019 getrennt nach den Altersgruppen. In der Gruppe der 80- bis 84-Jährigen lag die Sterberate im Peak in der 15. Kalenderwoche um $44 \%$ über dem Mittelwert der 


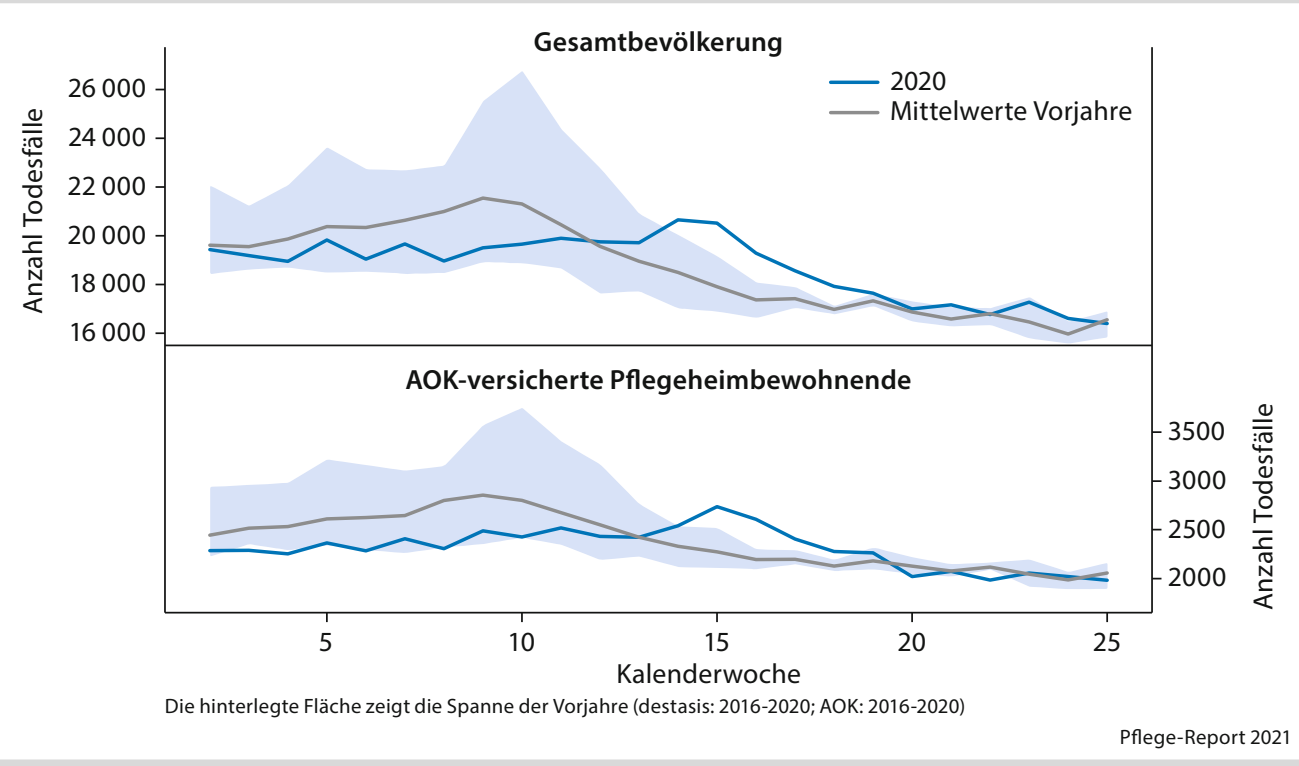

- Abb. 1.3 Sterblichkeit von vollstationär Pflegebedürftigen sowie in der Gesamtbevölkerung im Jahr 2020 im Vergleich zum Mittelwert der Vorjahre 2016-2019. (Quelle: AOK-Routinedaten 2016-2020; destatis 2016-2020)

Vorjahre. In den Gruppen der 85- bis 89-Jährigen wie auch bei den über 90-Jährigen war dieser Peak mit $17 \%$ bzw. $18 \%$ über dem Mittelwert weniger deutlich ausgeprägt, aber länger anhaltend.

Bei der Differenzierung nach Pflegegraden in - Abb. 1.5 zeigt sich, dass die Sterberaten unter den Bewohnenden mit Pflegegrad 2 oder 3 nur kurzzeitig über den Mittelwert der Vorjahre stiegen und ansonsten unter der maximalen Abweichung vom Mittelwert der Vorjahre verblieben. Unter den Bewohnenden mit Pflegegrad 4 - zwischen 33 und $38 \%$ aller Todesfälle entfielen in den Jahren 2015 bis 2019 auf diese Gruppe - lag die höchste Abweichung in der 15. Kalenderwoche bei $29 \%$ über dem Mittel der Vorjahre und blieb bis in die 19. Kalenderwoche über dem Mittelwert der Vorjahre. Noch ausgeprägter sind die Abweichungen im Pflegegrad 5, auf den in den Vorjahren zwischen 29 und $31 \%$ der Todesfälle entfielen. Der Anteil der Verstorbenen lag in der 15. Kalenderwoche um $37 \%$ über dem Mittelwert der Vorjahre und blieb bis zur 25. Kalenderwoche deutlich über dem Mittel- wert der Vorjahre. Eine Differenzierung der Bewohnenden nach Vorliegen einer dementiellen Erkrankung ${ }^{6}$ - zwischen 65 und $69 \%$ der Todesfälle entfielen in den Vorjahren auf Bewohnende mit Demenz - zeigt auch für dieses Klientel eine erhöhte Betroffenheit im Zeitraum der ersten Welle (• Abb. 1.6).

6 Bei Vorliegen einer im Krankenhaus gestellten oder mindestens zwei ambulant ärztlichen Diagnosen (F00, F01, F02, F03, F05.1, G23.1, G30, G31.0, G31.82) im aktuellen und dem letzten zurückliegenden Jahr. Für das Jahr 2020 liegen noch keine ambulant ärztlichen Diagnosen vor. Personen werden daher über Informationen der Jahre 2018/2019 den Kategorien Demenz ja/nein zugeordnet. Dies bedeutet, dass die Rate demenziell Erkrankter für 2020 tendenziell zu niedrig ausfällt, da Personen, die eine Demenz zu Beginn des Jahres neu entwickelt haben bzw. für die keine Vorjahresinformationen vorliegen, nicht erfasst werden können. Für das Jahr 2015 lagen keine Vorjahresinformationen vor, sodass das Vorliegen einer Demenz nur für die Jahre 2016 bis 2020 bestimmt werden konnte. 


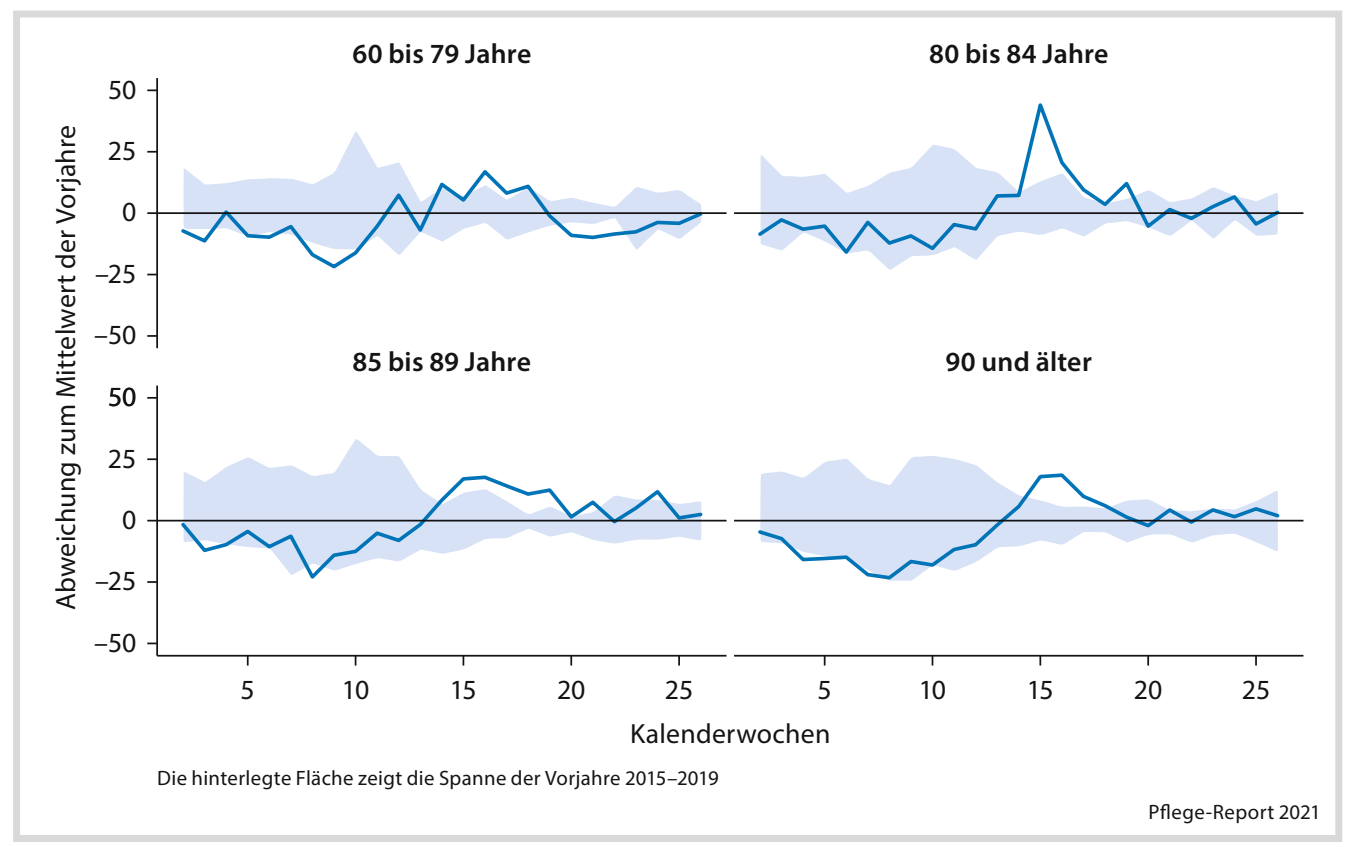

- Abb. 1.4 Sterblichkeit von vollstationär Pflegebedürftigen nach Altersgruppen im Jahr 2020 im Vergleich zum Mittelwert der Vorjahre 2015-2019, in \%. (Quelle: AOK-Routinedaten 2015-2020)

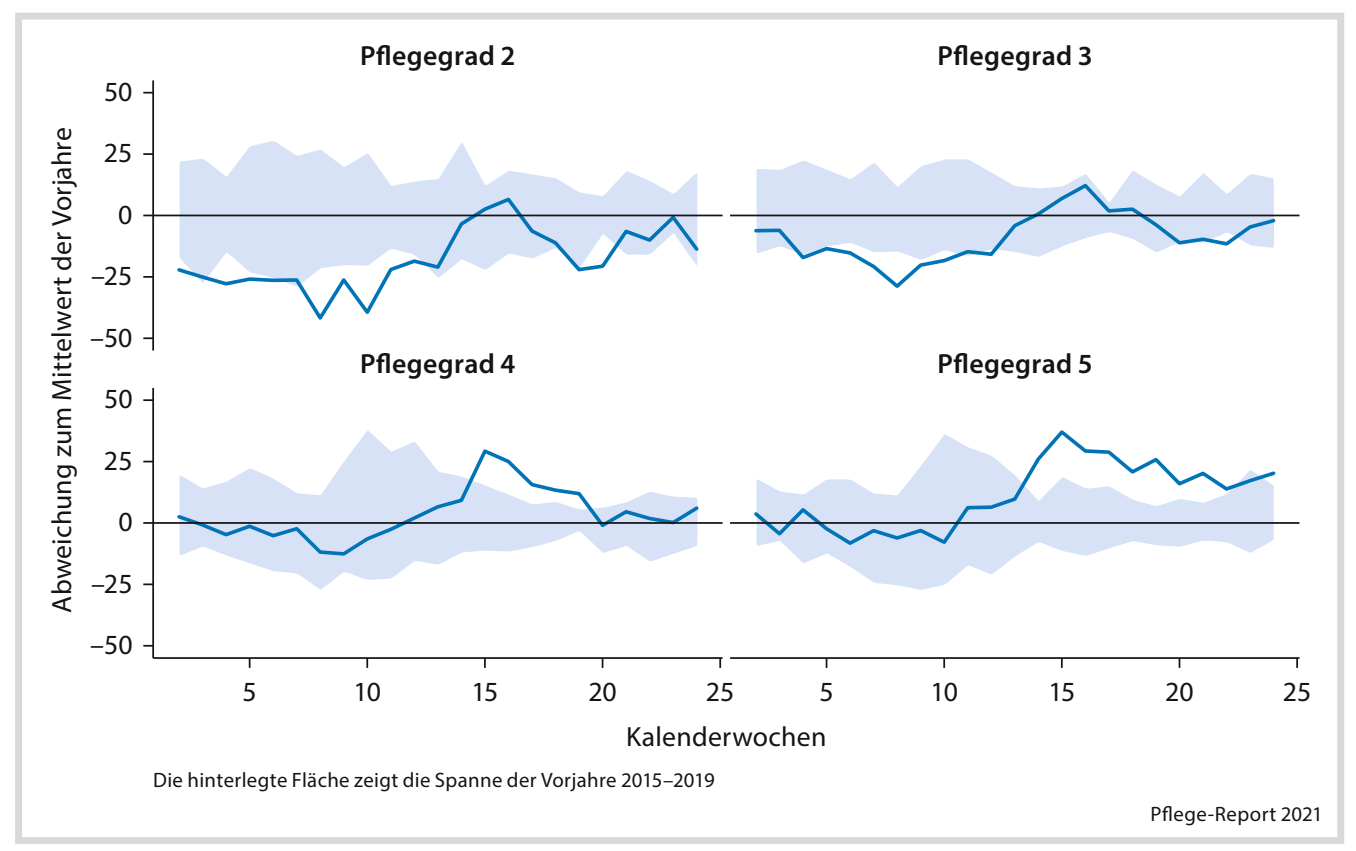

- Abb. 1.5 Sterblichkeit von vollstationär Pflegebedürftigen nach Pflegegraden im Jahr 2020 im Vergleich zum Mittelwert der Vorjahre 2015-2019, in \%. (Quelle: AOK-Routinedaten 2015-2020) 


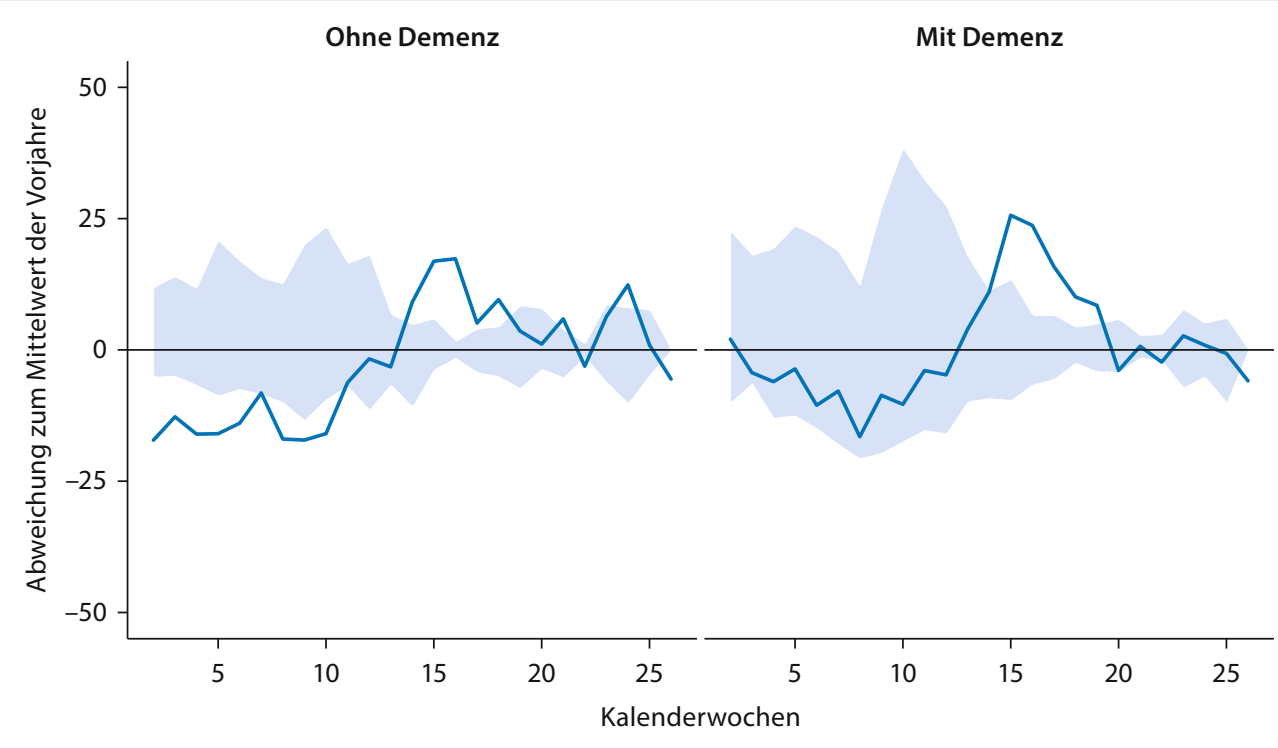

Die hinterlegte Fläche zeigt die Abweichungen vom Mittelwert der Vorjahre 2016-2020

Pflege-Report 2021

- Abb. 1.6 Sterblichkeit von vollstationär Pflegebedürftigen nach Demenz im Jahr 2020 im Vergleich zum Mittelwert der Vorjahre 2015-2019, in \%. (Quelle: AOK-Routinedaten 2016-2020)

\subsubsection{Pflegeheimbewohnende mit COVID-19 im Krankenhaus}

Für die erste COVID-Welle konnten ferner Krankenhausabrechnungsinformationen ausgewertet werden. Eingeflossen in die Analysen sind abgeschlossene Krankenhausfälle. Fokussiert wird im Folgenden der Zeitraum zwischen der 11. Kalenderwoche - hier rief die WHO den Pandemiezustand aus - und der 20. Kalenderwoche, in der bereits wieder eine bundesweite Inzidenz von täglich lediglich rund 600 Fällen und ein R-Wert von 0,88 erreicht waren. Ob die COVID-19 Infektion, angezeigt durch eine gesicherte Diagnose „U07.1 - COVID-19“, der Auslöser der Krankenhauseinweisung war oder erst während des Aufenthalts erworben wurde, kann auf Grundlage der Daten nicht differenziert werden. Verlegungen in andere Krankenhäuser wurden zu einem COVID-Fall zusammengefasst. Hat- te der Versicherte mehrere Krankenhausfälle mit einer COVID-Diagnose, geht er nur einmal in die hiesigen Analysen ein. Die Analysen sind wiederum nur bezogen auf Versicherte $a b$ 60 Jahren. - Abb. 1.7 fasst zusammen, dass innerhalb des Zeitraums der Kalenderwochen 11 bis 20 jeder fünfte COVID-Krankenhausaufenthalt $(21,7 \%)$ bei über 60-Jährigen und fast jeder dritte Todesfall im Krankenhaus mit Vorliegen einer COVID-Diagnose (31,6\%) auf einen Pflegeheimbewohner entfiel. Dies bedeutet, dass $43 \%$ der Pflegeheimbewohnenden mit COVID-Diagnose im Krankenhaus in diesem Zeitraum mit oder an COVID-19 verstorben sind, während es bei den übrigen Patientinnen und Patienten lediglich $26 \%$ waren (- Abb. 1.9).

Mehr als der Hälfte der Pflegeheimbewohnenden $(53 \%)$, bei denen während des Krankenhausaufenthalts eine gesicherte COVIDDiagnose dokumentiert wurde, waren 85 Jahre und älter. Die übrigen betroffenen Pati- 


\section{Anteil an KH-Fällen mit COVID}

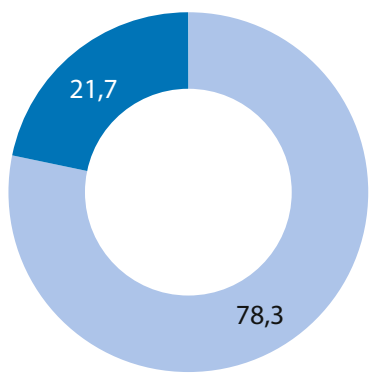

Anteil an Todesfällen im KH mit COVID

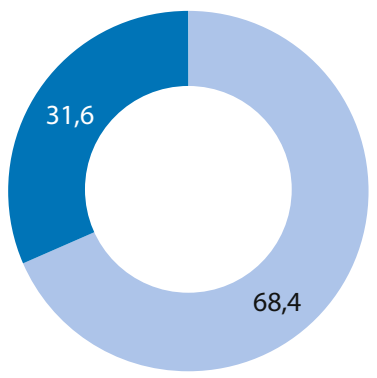

Vollstationär Pflegebedürftige

Versicherte (exkl. vollstationär Pflegebedürftige)

Pflege-Report 2021

- Abb. 1.7 Anteil Krankenhaus- und Todesfälle im Krankenhaus ( $\geq 60$ Jahre), die auf vollstationär Pflegebedürftige entfallen, bei denen eine gesicherte COVIDDiagnose dokumentiert wurde, in \%. (Quelle: AOK-Routinedaten 2020)

enten waren deutlich jünger (• Abb. 1.8). Der Anteil derjenigen, die mit oder an der COVID-Diagnose im Krankenhaus verster- ben, ist bei vollstationär Pflegebedürftigen im Vergleich zu Patienten der gleichen Altersgruppe, die nicht oder ambulant pflegebedürftig sind, deutlich erhöht (- Abb. 1.9). Dies betrifft vor allen Dingen die jüngeren Altersjahrgänge. Während $33 \%$ der 60 - bis 64-Jährigen Pflegeheimbewohnenden verstarben, lag die Rate bei den übrigen Patienten bei $10 \%$. Die Sterbewahrscheinlichkeiten der beiden Gruppen nähern sich mit steigendem Alter an: Bei den 80- bis 84-Jährigen verstarben $46 \%$ der Pflegeheimbewohnenden, bei den übrigen COVID-Patienten im Krankenhaus waren es $31 \%$. Bei den über 90-Jährigen liegt der Anteil Verstorbener in beiden Gruppen mit 48 und $44 \%$ bereits nah beieinander.

Schaut man auf die Sterbewahrscheinlichkeit differenziert nach Pflegegrad und dem Vorliegen einer Demenz (• Abb. 1.10), zeigt sich innerhalb der Grade ein relativ homogenes Bild bzw. keine eindeutige Tendenz, dass der Anteil Verstorbener mit steigendem Pflegegrad zunimmt. Personen mit demenzieller Erkrankung sind hingegen mit einem Anteil von $46 \%$ deutlich häufiger verstorben als Personen ohne Demenz mit einem Anteil von $38 \%$. Die Ergebnisse weisen auf die erhebliche Vulnerabilität der Pflegeheimbewohnenden hin, die aufgrund ihrer erhöhten Multimorbidität - zu den Determinanten von Pflegebedürftigkeit siehe Blüher et al. im gleichen Band - deutlich überproportional von die Prognose verschlechternden Vorerkrankungen wie Herz-Kreislauf-Erkrankungen, Diabetes,

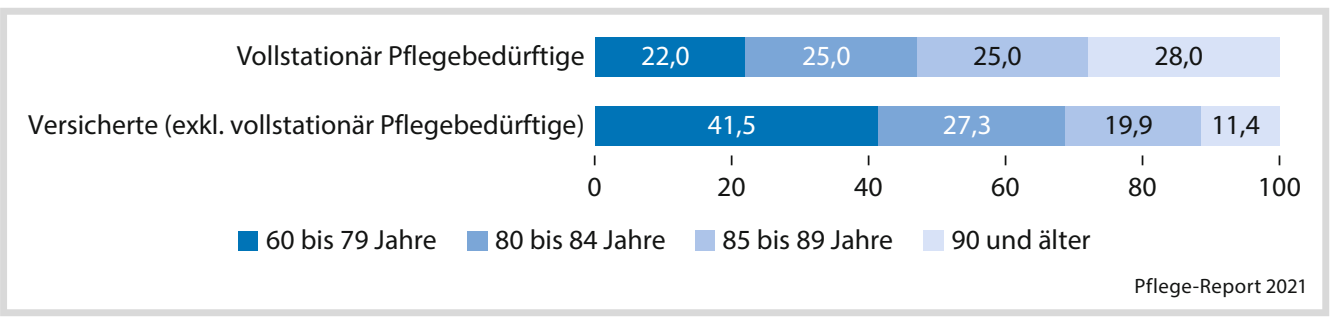

- Abb. 1.8 Krankenhausfälle ( $\geq 60$ Jahre), bei denen eine gesicherte COVID-Diagnose dokumentiert wurde, nach Altersgruppen, in \%. (Quelle: AOK-Routinedaten 2020) 


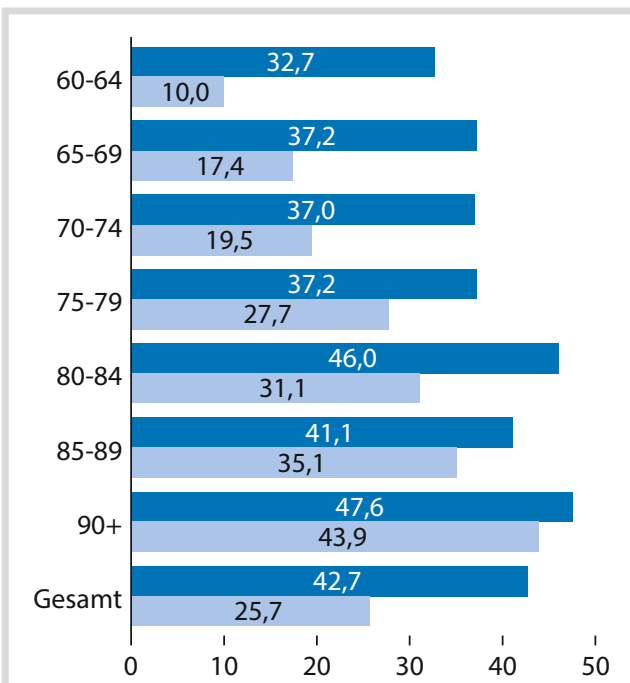

Vollstationär Pflegebedürftige

Versicherte (exkl. vollstationär Pflegebedürftige)

Pflege-Report 2021

Abb. 1.9 Anteil Verstorbene im Krankenhaus ( $\geq 60$ Jahre), bei denen eine gesicherte COVID-Diagnose dokumentiert wurde, nach Altersgruppen, in \%. (Quelle: AOK-Routinedaten 2020)

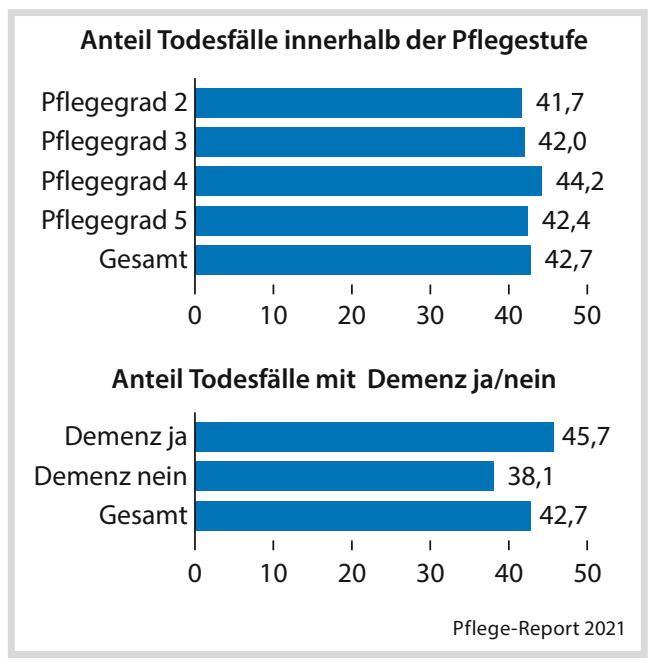

- Abb. 1.10 Anteil verstorbene vollstationär Pflegebedürftige im Krankenhaus ( $\geq 60$ Jahre), bei denen eine gesicherte COVID-Diagnose dokumentiert wurde, nach Pflegegraden und Demenz, in \%. (Quelle: AOK-Routinedaten 2020)
COPD oder Demenz (RKI 2021c) betroffen sind.

\subsubsection{Auswirkung der COVID- Pandemie auf die Krankenhausversorgung der Pflegeheimbewohnenden}

Ab Mitte März 2020 waren die Krankenhäuser angehalten, alle planbaren und elektiven Behandlungsanlässe $\mathrm{zu}$ verschieben (Mostert et al. 2021). Parallel dazu trat bereits mit dem COVID-19-Krankenhausentlastungsgesetz

Ende März der Krankenhausrettungsschirm in Kraft. Dies spiegelt sich auch in den Krankenhausaufenthalten der Stichprobe der Pflegeheimbewohnenden wider, die nach der 10. Kalenderwoche insgesamt erheblich zurückgingen: Im Vergleich zur 10. Kalenderwoche reduzierte sich der Anteil der Aufnahmen zur 12. Kalenderwoche um rund ein Drittel. Abb. 1.11 zeigt den Rückgang der Krankenhausaufnahmen gegenüber den Vorjahren.

Der Blick auf die Hauptdiagnosen der Krankenhausfälle nach der internationalen Klassifikation (ICD) gibt einen Einblick in das behandelte Erkrankungsspektrum. Die Herzinsuffizienz (I50.-) stellte im Zeitraum der Kalenderwochen 11 bis 20 in den Jahren 2015 bis 2019 den weitaus häufigsten dokumentierten Behandlungsanlass dar, gefolgt von der Pneumonie (Pneumonie, Erreger nicht näher bezeichnet J18.-) und der Hüftfraktur (Fraktur des Femurs S72.-). • Abb. 1.12 zeigt auf, in welchem Umfang die Krankenhausfallzahlen differenziert nach diesen häufigsten Behandlungsanlässen zurückgegangen sind. Deutlich wird, dass einerseits die Fallzahlen 2020 teilweise erheblich sanken, sich anderseits aber auch das Behandlungsspektrum verändert hatte. Hervorzuheben sind die Rückgänge bei sturzassoziierten Versorgungsanlässen wie Fraktur des Femurs (Rückgang 


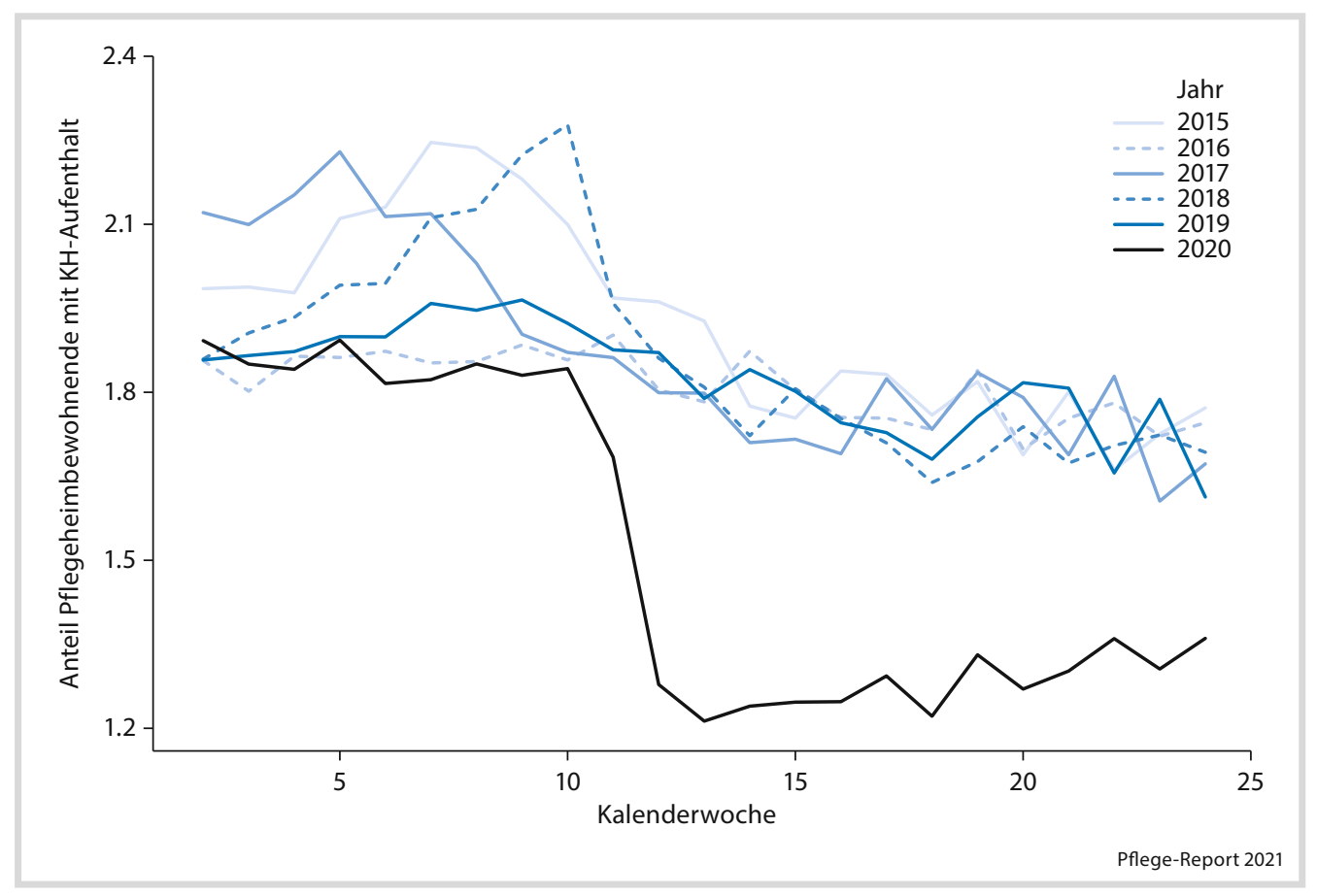

- Abb. 1.11 Anteil Pflegeheimbewohnender ( $\geq 60$ Jahre) mit Krankenhausaufenthalt 2015-2020, in \%. (Quelle: AOKRoutinedaten 2015-2020)

um $8 \%$ ) und intrakranieller Verletzung (Rückgang um $18 \%$ ). Ebenso wirft die Veränderungen der im Kontext der sogenannten ambulant-sensitiven Krankenhausdiagnosen $(\mathrm{ASK})^{7}$ zu verortenden Hauptdiagnosen Herzinsuffizienz (I50.-) - hier ist die Zahl der Fälle 2020 um $31 \%$ eingebrochen - oder Chro-

7 Unter ambulant-sensitiven Hospitalisierungen werden Krankenhauseinweisungen gefasst, die - so die These - durch ,Vorsorge oder rechtzeitige Intervention im ambulanten Sektor" nicht erforderlich wären. Sundmacher und Schüttig (2015) haben nach USamerikanischem Vorbild einen spezifischen deutschen Katalog ambulant-sensitiver Behandlungsanlässe im Krankenhaus (ASK) entwickelt. Da nicht für das hochaltrige Klientel und das Versorgungssetting Heim entwickelt, ist die Übertragung auf Pflegeheimbewohnende sicherlich eingeschränkt. Aktuell entwickelt die Universität Witten/Herdecke im Rahmen eines durch den Innovationsfonds geförderten Projekts eine Liste vermeidbarer Krankenhausbehandlungen für Heimbewohnende (PSK). nisch Obstruktive Lungenkrankheit (COPD) mit einem Rückgang von 39\% Fragen auf. Sind durch die Pandemie aufgrund der verminderten Mobilität der Bewohnerinnen und Bewohner Stürze „vermieden“ worden oder durch ggf. dann doch erfolgte ambulante Behandlung ggf. vermeidbare Krankenhausfälle verhindert worden oder zeigen die Ergebnisse auch eine Unterversorgung der Pflegeheimbewohnenden? Letzteres wäre am Beispiel der Fallzahlen des Hirninfarktes (I63.-) weitergehend zu untersuchen, die in der Stichprobe der hier analysierten Bewohnenden um $20 \%$ eingebrochen sind. Drogan et al. 2020 haben für die Fallzahlreduktion für die Notfallindikation (akuter Hirngefäßverschluss mit über $24 \mathrm{~h}$ anhaltender neurologischer Funktionsstörung oder Nachweis einer durch Durchblutungsstörung ausgelösten Hirnläsion) dargelegt, dass der Rückgang der Schlaganfall-Behandlungszahlen nicht aus einer Überforderung des Ver- 


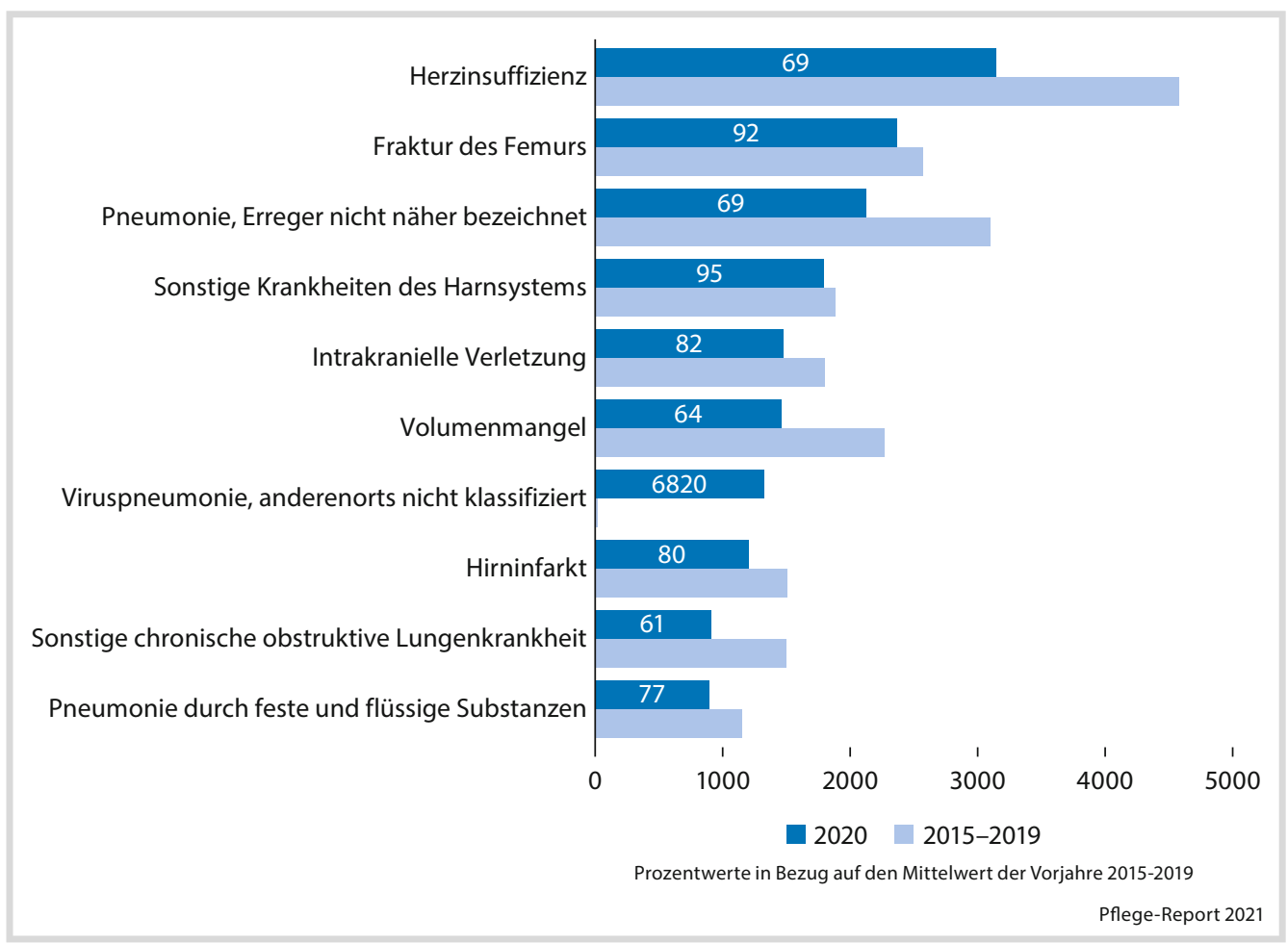

- Abb. 1.12 Krankenhausfälle 2020 nach den zehn häufigsten Hauptdiagnosen (nach ICD-Kapiteln) im Vergleich zum Mittelwert der Vorjahre 2015-2019, in \%

sorgungssystems Krankenhaus resultiert und vermuten, dass die Ursachen darin liegen, dass die Bevölkerung einen nicht für unbedingt nötig gehaltenen Krankenhausaufenthalt vermied sowie Verzögerungen bei den Einweisungen älterer Menschen aufgrund sozialer Isolation während der Pandemie auftraten. Dies wirft für das Versorgungssetting Pflegeheim bezüglich der ärztlichen Betreuung - aber auch der Initiierung dieser durch die Pflegefachkräfte - erhebliche Fragen auf, denn qualifiziertes Personal sollte wissen, dass Betroffene bei Schlaganfallsymptomen umgehend ärztliche Behandlung erhalten sollten, auch in Hochphasen der Pandemie und im Lockdown.

\subsection{Diskussion und Ausblick}

Schon vor der Pandemie zeigten sich deutliche Defizite hinsichtlich des Zusammenlebens so vieler hochbetagter, meist schwer kranker Menschen: Die Personaldecke in der Pflege ist viel zu dünn (Rothgang et al. 2020), die Qualifikation des Personals ist angesichts der Krankheitslage nicht mehr adäquat (vgl. Stemmer in diesem Band), die Patientensicherheitskonzepte sind unzureichend (Hölscher et al. 2014) und die fachärztliche Betreuung ist für die meisten Fachgruppen unterproportional (Schulz et al. 2020).

Die Corona-Krise deckt diese Schwächen des stationären Pflegesystems auf Kosten der Pflegebedürftigen, ihrer Angehörigen und des pflegerischen Personals nun konsequent auf. Dieser Beitrag legt erste Befunde aus dem 
Kontext des durch den GKV-Spitzenverband geförderten Forschungsprojekts Covid-Heim vor, das die Charité - Universitätsmedizin Berlin in Kooperation mit dem Wissenschaftlichen Institut der AOK (WIdO) durchführt. Er beschränkt sich dabei auf die Situation der Pflegebedürftigen im Heim in der ersten Pandemiewelle von März bis Mai 2020 mit Ergebnissen zur allgemeinen Sterblichkeit sowie zur Sterblichkeit bei im Krankenhaus behandelter COVID-Erkrankung und auf Befunde zu den Veränderungen in der Krankenhausbehandlung von Pflegeheimbewohnerinnen und -bewohnern. Die Fakten zusammengefasst: Die Sterblichkeit von vollstationär Pflegebedürftigen vor der ersten Corona-Welle des Jahres 2020 war geringer als in den Vorjahren 2015, 2017 und 2018 mit ihren ausgeprägten Grippewellen, jedoch wendete sich das Blatt mit der 14. Kalenderwoche 2020. Bereits drei Wochen nach dem Start des Lockdowns am 16. März lag der Anteil der Verstorbenen in den Heimen verglichen zum Mittel der Vorjahre um $20 \%$ höher. Und jeder fünfte COVID-bedingte Krankenhausaufenthalt und fast jeder dritte COVID-Todesfall im Krankenhaus entfiel auf eine vollstationär versorgte pflegebedürftige Person. Die Ergebnisse zum massiven Rückgang der Krankenhausaufnahmen von Pflegebedürftigen aus den Heimen um rund ein Drittel zwischen der 10. und 12. Kalenderwoche des Jahres 2020 könnten auf eine mögliche gesundheitliche Unterversorgung der Pflegeheimbewohnenden in der Periode des ersten Lockdowns hindeuten.

Als Fazit dieser nachgewiesenen Problemfelder ergeben sich Ableitungen sowohl für künftigen Forschungsbedarf als auch für erste Handlungsbereiche. Deutlich wird mit der schnell steigenden Mortalität und den hohen Zahlen an verstorbenen Heimbewohnerinnen und -bewohnern, dass die direkten gesundheitlichen Auswirkungen auf die hochbetagte Klientel bereits in der Anfangsphase der Pandemie massiv waren (vgl. RKI 2021d). Noch gibt es Evidenzlücken, welchen Komorbiditäten und deren Zusammenspiel im Setting Pflegeheim welche Bedeutung zukommt, wenn es darum geht, den Tod der an COVID-19 erkrankten Heimbewohnenden zu erklären. Auch muss erforscht werden, ob und wie der Personalmangel, die psychische und physische Erschöpfung des Pflegepersonals und das eigene Krankheits- und Infektionsgeschehen beim Pflegepersonal zur Krisensituation in den Heimen beigetragen hat (vgl. Covid-Heim/1. Report 2021). Klar wird angesichts der Fakten ebenso, dass der Infektionsschutz für die pflegebedürftige Gruppe der im Heim Lebenden in der ersten Phase der Pandemie nicht ausreichte, um diese vor massenhaften Ansteckungen und der hohen Mortalität zu schützen (vgl. Schrappe et al. 2020a). Dringend muss nun untersucht werden, inwiefern die verbesserte Hygiene- und Schutzsituation (Masken, Schutzkleidung für Pflegende und Angehörige, Tests, Umbauten in den Heimen z. B. mit Plexiglaswänden, später: Impfen) während der zweiten Pandemiewelle ein Baustein der Infektionsprävention darstellte und welche dieser Schutzmaßnahmen zur Standardausstattung aller Heime zur Vermeidung von Infektionen bei künftig auftretenden viralen Gefährdungen gehören müssen.

Was auf keinen Fall noch einmal zur Gefährdungsvermeidung herangezogen werden darf, ist die generelle Isolierung alter Frauen und Männer von der Außenwelt und von ihren Angehörigen. Untersucht werden muss, wie Isolation, Kontaktsperren zu Angehörigen und die Einschränkung der Bewegungsfreiheit das Leben der Bewohnerinnen und Bewohner beeinflussten und welche technischen, baulichen, rechtlichen und personellen Veränderungen und Ressourcen benötigt werden, um zu vermeiden, dass sich eine solche Situation wiederholt. Noch fehlt es zudem an Evidenz zu den Langzeitwirkungen solcher Maßnahmen auf Gesundheit und Wohlbefinden der betagten Frauen und Männer im weiteren Lebensverlauf.

Weitere Forschungsfragen ergeben sich aus den oben dargestellten Ergebnissen zu den veränderten Krankenhausaufnahmen sowie dem Rückgang der Behandlungsfälle von Pflegeheimbewohnerinnen und -bewohnern im 
Krankenhaus in der ersten Welle der Pandemie. Übernahm das ambulante Versorgungssystem Behandlungen, die vorher stationär versorgt wurden? Wurden möglicherweise unnötige Krankenhauseinweisungen vermieden? Wurden eigentlich notwendige Behandlungen im Krankenhaus aus Sorge vor einer Infizierung nicht durchgeführt? Dies alles sind Forschungsfragen, die nicht nur generell, sondern auch konkret für die Situation der Pflegebedürftigen in der stationären Langzeitversorgung gestellt werden müssen.

Es sind die oben genannten Fragen, die im Rahmen des Projekts Covid-Heim in den kommenden Monaten weiter untersucht werden mit dem Ziel, die Situation der Heimbewohnerinnen und -bewohner, ihrer Angehörigen und der Pflegekräfte möglichst umfassend zu beschreiben, um so eine belastbare Evidenz für Veränderungen im Versorgungssetting der stationären Langzeitpflege zu erhalten.

\section{Literatur}

Asim M, Sathian B, Van Teijlingen E, Mekkodathil A, Subramanya SH, Simkhada P (2020) COVID-19 pandemic: public health implications in Nepal. Nepal $\mathbf{J}$ Epidemiol 10(1):817

Buonsenso D, Piano A, Raffaelli F, Bonadia N, Donati KDG, Franceschi F (2020) Novel coronavirus disease-19 pneumoniae: a case report and potential applications during COVID-19 outbreak. Eur Rev Med Pharmacol Sci 24:2776-2780

Bund-Länder-Beschlüsse (2020) Telefonkonferenz der Bundeskanzlerin mit den Regierungschefinnen und Regierungschefs der Länder am 13. Dezember. https://www.bundesregierung.de/

resource/blob/997532/

1827366/69441fb68435a7199b3d3a89bff2c0e6/ 2020-12-13-beschluss-mpk-data.pdf?download=1 . Zugegriffen: 14. Dez. 2020

Covid-Heim 1. Report (2021) Lehren aus der CoronaPandemie für Strukturentwicklungen im Versorgungssetting Pflegeheim. Ergebnisreport 1: Systemrelevant und stark belastet: Zum Befinden des Pflegepersonals in der Corona-Pandemie. http://www.charite.de

Cucinotta D, Vanelli M (2020) WHO declares COVID-19 a pandemic. Acta Bio Medica: Atenei Parmensis 91(1): 157

DGP - Deutsche Gesellschaft für Pflegewissenschaft e. V. (Hrsg) (2020) S1-Leitlinie - Soziale Teilhabe und
Lebensqualität in der stationären Altenhilfe unter den Bedingungen der COVID-19-Pandemie - Langfassung - AWMF-Registernummer 184-001. https:// www.awmf.org/leitlinien/aktuelleleitlinien.html. Zugegriffen: 24. Nov. 2020

Drogan D, Pfeilschifter W, Scholz K, Zacher J, Günster C (2020) Effekte des Covid-19-Lockdowns auf die stationäre Behandlung von Patienten mit Herzinfarkt, Schlaganfall und Hüftfraktur in Deutschland. In: Klauber J, Dormann F, Kuhlen R (Hrsg) Qualitätsmonitor 2020. Medizinisch wissenschaftliche Verlagsgesellschaft (MWV), Berlin, S 223-260

Hölscher U, Gausmann P, Haindl HH, Heidecke CD, Hübner NO, Lauer W, Lauterberg J, Skorning M, Thürmann P (2014) Patientensicherheit als nationales Gesundheitsziel: Status und notwendige Handlungsfelder für die Gesundheitsversorgung in Deutschland. Z Evid Fortbild Qual Gesundheitsw 108:6-14

Holt-Lunstad J, Smith TB, Layton JB (2010) Social relationships and mortality risk: a meta-analytic review. PLoS Med 7(7):e1000316. https://doi.org/10. 1371/journal.pmed.1000316

Hower KI, Pfaff H, Pförtner T-K (2020) Pflege in Zeiten von COVID-19: Onlinebefragung von Leitungskräften zu Herausforderungen, Belastungen und Bewältigungsstrategien. Pflege 33(4):207-218. https://doi. org/10.1024/1012-5302/a000752

Iaccarino G, Grassi G, Borghi C, Ferri C, Salvetti M, Volpe $M$ (2020) Age and multimorbidity predict death among COVID-19 patients: results of the SARS-RAS study of the Italian Society of Hypertension. Hypertension 76(2):366-372

Karagiannidis C, Mostert C, Hentschker C, Voshaar T, Malzahn J, Schillinger G, Klauber J, Janssens U, Marx G, Weber-Carstens S, Kluge S, Pfeifer M, Grabenhenrich L, Welte T, Busse R (2020) Case characteristics, resource use, and outcomes of 10021 patients with COVID-19 admitted to 920 German hospitals: an observational study. Lancet Respir Med 8(9):853-862, https://doi.org/10.1016/ S2213-2600(20)30316-7

Keller M Corona in Alten- und Pflegeheimen: „Die Pandemie hat uns kalt erwischt“. https://www.tagesspiegel. de/wissen/corona-in-alten-und-pflegeheimendie-angehoerigen-reagieren-zu-95-prozent-mitverstaendnis/25756662-2.html (Erstellt: 24. Apr. 2020). Zugegriffen: 3. Dez. 2020

Kessler E-M, Strumpen S, Kricheldorff C, Franke A, Pantel J, Gellert P (2020) „Partizipation und soziale Teilhabe älterer Menschen trotz Corona-Pandemie ermöglichen": Gemeinsames Statement der Sektionen für Geriatrische Medizin (II), Sozial- und verhaltenswissenschaftliche Gerontologie (III), Soziale Gerontologie und Altenhilfe (IV) der Deutschen Gesellschaft für Gerontologie und Geriatrie (DGGG e. V.) vom 24. Apr. 2020. https://www.dggg-online. de/fileadmin/aktuelles/covid-19/20200424_DGGG_ 
Statement_Sektionen_II_III_IV_Soziale_Teilhabe und_Partizipation.pdf, Zugegriffen:03. Dez. 2020

Mangiapane S, Zhu L, Czihal T, von Stillfried D (2021) Veränderung der vertragsärztlichen Leistungsinanspruchnahme während der COVID-Krise. https:// www.zi.de/fileadmin/images/content/Publikationen/ Trendreport_3_Leistungsinanspruchnahme_COVID_ final.pdf. Zugegriffen: 16. Febr. 2021

Mueller AL, McNamara MS, Sinclair DA (2020) Why does COVID-19 disproportionately affect the elderly? Preprints.org https://doi.org/10.20944/ preprints202004.0548.v1

Mostert C, Hentschker C, Scheller-Kreinsen D, Günster C, Malzahn J, Klauber J (2021) Auswirkungen der Covid-19-Pandemie auf die Krankenhausleistungen im Jahr 2020. In: Klauber J, Wasem J, Beivers A, Mostert C (Hrsg) Krankenhaus-Report 2021. Versorgungsketten - Der Patient im Mittelpunkt. Springer, Berlin Heidelberg, S 277-306

Panagiotou OA, Kosar CM, White EM, Bantis LE, Yang X, Santostefano CM, Mor V (2021) Risk factors associated with all-cause 30-day mortality in nursing home residents with $\mathrm{CO}$ VID-19. JAMA Intern Med 181(4):439-448, DOI:10.1001/jamainternmed.2020.7968

RKI - Robert Koch-Institut (2020) Täglicher Lagebericht des RKI zur Coronavirus-Krankheit-2019 (COVID-19) vom 11. März 2020. https://www.rki. de/DE/Content/InfAZ/N/Neuartiges_Coronavirus/ Situationsberichte/2020-03-11-de.pdf?_blob= publicationFile. Zugegriffen: 12. Jan. 2021

RKI - Robert Koch-Institut (2021a) Epidemiologischer Steckbrief zu SARS-CoV-2 und COVID-19 Stand: 18.3.2021. https://www.rki.de/DE/Content/InfAZ/N/ Neuartiges_Coronavirus/Steckbrief.html. Zugegriffen: 20. März 2021

RKI - Robert Koch-Institut (2021b) Täglicher Lagebericht des RKI zur Coronavirus-Krankheit-2019 (COVID-19) 20.03.2021 - aktualisierter Stand für Deutschland. https://www.rki. de/DE/Content/InfAZ/N/Neuartiges_Coronavirus/ Situationsberichte/Maerz_2021/2021-03-20-de. pdf?_blob=publicationFile. Zugegriffen: 20. März 2021

RKI - Robert Koch-Institut (2021c) Epidemiologisches Bulletin 2/2021 - Beschluss der STIKO zur 1. Aktualisierung der COVID-19-Impfempfehlung. https:// www.rki.de/DE/Content/Infekt/EpidBull/Archiv/ 2021/Ausgaben/02_21.pdf?_blob=publicationFile. Zugegriffen: 15. März 2021
RKI - Robert Koch-Institut (RKI) (2021d) Journal of Health Monitoring (JoHM S4/2021): Zur Situation älterer Menschen in der Anfangsphase der COVID-19-Pandemie: Ein Scoping Review. www.rki.de/johm-covid19. Zugegriffen: 27. März 2021

Rothgang H, Cordes J, Fünfstück M, Heinze F, Kalwitzki T, Stolle C (2020) Abschlussbericht im Projekt Entwicklung und Erprobung eines wissenschaftlich fundierten Verfahrens zur einheitlichen Bemessung des Personalbedarfs in Pflegeeinrichtungen nach qualitativen und quantitativen Maßstäben gemäß $\S 113 \mathrm{c}$ SGB XI (PeBeM). https://www.gs-qsa-pflege.de/wpcontent/uploads/2020/09/Abschlussbericht_PeBeM. pdf. Zugegriffen: 30. März 2021

Schrappe M, François-Kettner H, Gruhl M, Hart D, Knieps F, Manow P, Pfaff H, Püschel K, Glaeske G (2020a) Thesenpapier 3: Die Pandemie durch SARSCoV-2/CoViD-19 - Strategie: Stabile Kontrolle des Pandemiegeschehens, Prävention: Risikosituationen verbessern, Bürgerrechte: Rückkehr zur Normalität. Köln Berlin Bremen Hamburg. http://www.metthias. schrappe.com/index.htm. Zugegriffen: 27. März 2021

Schrappe M, François-Kettner H, Gruhl M, Hart D, Knieps F, Manow P, Pfaff H, Püschel K, Glaeske G (2020b) Thesenpapier 6, Teil 6.1: Epidemiologie. Die Pandemie durch SARS-CoV-2/CoViD-19, Zur Notwendigkeit eines Strategiewechsels. Köln Berlin Bremen Hamburg, 22.11. Monit Versorgungsforsch 13:76-92. https://doi.org/10.24945/MVF.06.20.1866-0533.2267

Schulz M, Tsiasioti C, Czwikla J, Schwinger A, Gand D, Schmidt A, Schmiemann G, Wolf-Ostermann K, Rothgang H (2020) Claims data analysis of medical specialist utilization among nursing home residents and community-dwelling older people. BMC Health Serv Res 20(1):690. https://doi.org/10.1186/s12913020-05548-0

Simonetti A, Pais C, Jones M, Cipriani MC, Janiri D, Monti L, Landi F, Bernabei R, Liperoti R, Sani G (2020) Neuropsychiatric Symptoms in Elderly With Dementia During COVID-19 Pandemic: Definition, Treatment, and Future Directions. Front Psychiatry 11:579842. DOI: 10.3389/fpsyt.2020.579842

Sundmacher L, Schüttig W (2015) Which hospitalisations are ambulatory care-sensitive, to what degree, and how could the rates be reduced? Results of a group consensus study in Germany. Health Policy 11:1415-1423 
Open Access Dieses Kapitel wird unter der Creative Commons Namensnennung 4.0 International Lizenz (http:// creativecommons.org/licenses/by/4.0/deed.de) veröffentlicht, welche die Nutzung, Vervielfältigung, Bearbeitung, Verbreitung und Wiedergabe in jeglichem Medium und Format erlaubt, sofern Sie den/die ursprünglichen Autor(en) und die Quelle ordnungsgemäß nennen, einen Link zur Creative Commons Lizenz beifügen und angeben, ob Änderungen vorgenommen wurden.

Die in diesem Kapitel enthaltenen Bilder und sonstiges Drittmaterial unterliegen ebenfalls der genannten Creative Commons Lizenz, sofern sich aus der Abbildungslegende nichts anderes ergibt. Sofern das betreffende Material nicht unter der genannten Creative Commons Lizenz steht und die betreffende Handlung nicht nach gesetzlichen Vorschriften erlaubt ist, ist für die oben aufgeführten Weiterverwendungen des Materials die Einwilligung des jeweiligen Rechteinhabers einzuholen. 